\title{
Transplante de membrana amniótica para tratamento do pterígio recidivado
}

\author{
Amniotic membrane transplantation for recurrent pterygium treatment
}

\author{
Ligia Fernanda Bruni ${ }^{1}$ \\ Silvana Artioli Schellini ${ }^{2}$ \\ Elisângela Jaqueta ${ }^{3}$ \\ Amélia Kamegasawa ${ }^{4}$ \\ Carlos Roberto Padovani ${ }^{5}$
}

\begin{tabular}{|l|}
\hline RESUMO \\
\hline Objetivo: Avaliar a efetividade do uso da membrana amniótica para o \\
tratamento do pterígio recidivado. Métodos: Foram operados 24 pacientes \\
(27 olhos) portadores de pterígio recidivado, 7 dos quais com simbléfaro \\
associado usando-se enxerto de membrana amniótica preservada. Foram \\
analisados parâmetros como idade, sexo, olho acometido, localização do \\
pterígio, número de cirurgias anteriores, bem como complicações pós- \\
operatórias e recidiva, em período de seguimento de 6 meses. Resultados: \\
$58,3 \%$ dos pacientes eram do sexo masculino e $58,3 \%$ encontravam-se na \\
faixa etária de 40 a 59 anos. Todos eram portadores de pterígio recidivado \\
e $48,1 \%$ deles já tinham feito pelo menos duas cirurgias anteriores. Os \\
pacientes foram acompanhados por 6 meses. Houve recidiva do pterígio \\
em $18,5 \%$ dos casos e em 11,1\%, formação de granuloma. Conclusões: \\
Otratamento do pterígio recidivado com transplante demembrana amniótica \\
é um procedimento relativamente simples, com baixo índice de complica- \\
ções e que traz bons resultados. \\
\hline
\end{tabular}

Descritores: Curativos biológicos/utilização; Pterígio/cirurgia; Âmnio/transplante; Recidiva; Procedimentos cirúrgicos oftalmológicos
Trabalho realizado na Faculdade de Medicina de Botucatu, apresentado como Poster no Congresso Brasileiro de Prevenção de Cegueira - 2000.

' Residente da Disciplina de Oftalmologia do Departamento de Oftalmologia, Otorrinolaringologia e Cirurgia de Cabeça e Pescoço da Faculdade de Medicina de Botucatu - Universidade Estadual Paulista.

2 Professor Livre-docente do Departamento de Oftalmologia, Otorrinolaringologia e Cirurgia de Cabeça e Pescoço - Disciplina de Oftalmologia da Faculdade de Medicina de Botucatu - Universidade Estadual Paulista.

${ }^{3}$ Ex-Residente da Disciplina de Oftalmologia do Departamento de Oftalmologia, Otorrinolaringologia e Cirurgia de Cabeça e Pescoço da Faculdade de Medicina de Botucatu - Universidade Estadual Paulista.

${ }^{4}$ Professor Assistente Doutor do Departamento de Oftalmologia, Otorrinolaringologia e Cirurgia de Cabeça e Pescoco - Disciplina de Oftalmologia da Faculdade de Medicina de Botucatu - Universidade Estadual Paulista. ${ }_{5}^{5}$ Professor Titular do Departamento de Bioestatística Instituto de Biociências - Universidade Estadual Paulista - Botucatu.

Endereco para correspondência: Silvana Artioli Schellini - DEP. OFT/ORL/CCP - Faculdade de Medicina de Botucatu - UNESP - Botucatu (SP) CEP 18618-000. E-mail: sartioli@fmb.unesp.br

Nota Editorial: Pela análise deste trabalho e por sua anuência sobre a divulgação desta nota, agradecemos ao Dr. Hamilton Moreira.

\section{INTRODUÇÃO}

O uso de membrana amniótica (MA) tem conquistado espaço cada vez maior no tratamento das afecções da superfície ocular, como na síndrome de Stevens-Johnson ${ }^{(1)}$, no penfigóide cicatricial ${ }^{(1)}$, nas queimaduras químicas e térmicas ${ }^{(2-3)}$, em simbléfaro de várias etiologias ${ }^{(4)}$, em úlceras corneanas $^{(5)}$ e no pterígio recidivado ${ }^{(6-7)}$.

A MA atua beneficiando a epitelização, uma vez que facilita a migração e a adesão das células epiteliais basais, previne a apoptose e restaura o fenótipo epitelial ${ }^{(8)}$.

Além disso, é considerada imunologicamente inerte, tornando-se uma atraente opção para enxertos ${ }^{(9)}$.

Shimazaki et al. ${ }^{(6)}$, utilizaram o transplante de MA preservada juntamente com auto-transplante de limbo em pacientes com pterígio recidivado associado a simbléfaro em 4 pacientes e observaram recidiva do pterígio em apenas 1 caso.

O presente trabalho teve por objetivo observar a efetividade do uso da MA preservada para o tratamento de pterígios recidivados.

\section{MÉTODOS}

Foi realizado estudo prospectivo em 24 pacientes ( 27 olhos) portadores de pterígio recidivado, associado ou não a simbléfaro, operados na Facul- 
dade de Medicina de Botucatu- UNESP, nos quais se utilizou MA preservada. Os pacientes foram consultados e consentiram com o procedimento.

\section{Técnica de obtenção e preservação da MA}

A MA foi obtida de parto cesárea, de gestantes com exames sorológicos negativos para HIV, Lues, Hepatite B e hematológicos normais e sem patologias sistêmicas associadas. Após a dequitação, foi separada a MA do córion, em ambiente estéril. Em seguida, a membrana foi lavada com soro fisiológico e solução contendo cefalosporina $(1 \mathrm{~g})$ e gentamicina $(40 \mathrm{mg})$ e mergulhada por alguns segundos em solução de Dulbecco modificado Eagle (Cultilab-Campinas, SP). Após, a MA foi colocada sobre papel de filtro de nitrocelulose (MILIPORE-S-PAK 41-São Paulo, SP), cortados na forma de discos de $47 \mathrm{~mm}$ de diâmetro, com a face epitelial voltada para cima. Os discos foram dobrados e introduzidos em frascos contendo o meio Dulbecco modificado Eagle e glicerol (1:1) e conservada a $-80^{\circ} \mathrm{C}$. Antes da utilização, a MA foi reidratada em soro fisiológico até que sua coloração voltasse a ser como antes da preservação.

\section{Técnica cirúrgica}

As cirurgias foram realizadas por 2 dos autores (LFB e EJ). Após antissepsia com clorohexexidina alcóolica, colocação de campos estéreis e blefarostato, foi feita anestesia subconjuntival no local da exerese, com agulha de insulina, usando Xylocaína 2,0\% com vasoconstrictor. Procedeu-se a excisão da cabeça do pterígio com lâmina 15 e ressecção da cabeça e corpo com tesoura; quando havia simbléfaro associado, o tecido fibrótico foi ressecado.

A conjuntiva foi retrocedida e fixada na episclera com fio de Vycryl 7-0 (Ethicon), deixando o leito escleral próximo ao limbo livre. A membrana já reidratada foi, colocada sobre o leito cruento, com a face epitelial voltada para cima. A membrana não foi suturada, sendo estabilizada no leito com a colocação de lente de simbléfaro com orifício central. Realizou-se tarsorrafia com fio de seda 6-0.

Após o procedimento os pacientes ficaram com curativo oclusivo por 24 horas e iniciou-se o uso de colírio de dexametasona e cloranfenicol (Dexafenicol ${ }^{\mathbb{}}$ - Allergan/Frumtost Ltda) $4 \mathrm{x} /$ dia até o $15^{\circ}$ dia de pós-operatório (PO).
A tarsorrafia foi removida no $7^{\circ} \mathrm{PO}$ e a lente de simbléfaro foi mantida até o $15^{\circ} \mathrm{PO}$.

\section{Parâmetros avaliados}

- Relativos aos pacientes: idade, sexo, olho acometido, localização do pterígio, número de cirurgias anteriores.

- Relativas ao procedimento efetuado: complicações pós-operatórias e recidiva. Como critério para classificar recidiva consideramos a presença de tecido fibroblástico conjuntival ultrapassando o limbo e invadindo a córnea, com vascularização local.

O tempo de seguimento foi de 6 meses e os pacientes foram avaliados no $7^{\circ}, 15^{\circ}, 30^{\circ}, 60^{\circ}, 90^{\circ}$ e $180^{\circ}$ dias de PO. Os dados foram submetidos a análise estatística descritiva envolvendo a distribuição de freqüência dos dados.

\section{RESULTADOS}

Os dados relativos às características dos pacientes operados estão apresentados na tabela 1.

A freqüência das alterações oculares observadas no PO encontra-se na tabela 2.

O procedimento cirúrgico foi realizado com facilidade em todos os casos. Observou-se, sempre nos primeiros dias de

\begin{tabular}{|lccc|}
\hline \multicolumn{4}{|c}{$\begin{array}{c}\text { Tabela 1. Características dos portadores de pterígio recidivado } \\
\text { submetidos a cirurgia com uso de membrana amniótica }\end{array}$} \\
& Variável & № Pacientes & $\%$ \\
Sexo & Masculino & 14 & 58,3 \\
& Feminino & 10 & 41,7 \\
Faixa etária & $\leq 20$ & 01 & 41,6 \\
& $20-39$ & 06 & 25,0 \\
& $40-59$ & 14 & 58,3 \\
Olho & $\geq 60$ & 03 & 12,5 \\
& Direito & 09 & 33,3 \\
& Esquerdo & 12 & 44,4 \\
Localização & Ambos & 03 & 11,1 \\
& Nasal & 21 & 77,7 \\
Cirurgia prévia & Temporal & 03 & 11,1 \\
& Nasal + Temporal & 03 & 11,1 \\
& 1 & 14 & 51,8 \\
& 2 & 09 & 33,3 \\
& 3 & 04 & 14,8 \\
\hline
\end{tabular}

\begin{tabular}{|c|c|c|c|c|c|c|}
\hline Alterações & 7 & 15 & 30 & 90 & 180 & $\begin{array}{l}\text { Total } \\
(\%)\end{array}$ \\
\hline Perda da MA & $2(7,4)$ & 0 & 0 & 0 & 0 & $2(7,4)$ \\
\hline Reabsorção da MA & $2(7,4)$ & $11(40,7)$ & $12(44,4)$ & 0 & 0 & $25(92,6)$ \\
\hline Granuloma & $1(4,6)$ & $2(7,4)$ & 0 & 0 & 0 & $3(11,1)$ \\
\hline
\end{tabular}


PO, a presença de secreção esbranquiçada em quantidade moderada, sem sinais infecciosos.

A membrana estava presente no $7^{\circ}$ PO na maioria dos pacientes, estando ausente em dois pacientes por ter sido absorvida e em outros dois, por ter sido removida pelo próprio paciente. Na maior parte dos casos a membrana foi absorvida entre o $15^{\circ}$ e o $30^{\circ} \mathrm{PO}$.

Houve formação de granuloma em 3 olhos: um logo no $7^{\circ}$ $\mathrm{PO}$ e outros dois no $15^{\circ}$. Esta complicação foi solucionada com a exerese cirúrgica dos granulomas.

Não houve recidiva de simbléfaro e houve recidiva do pterígio em 5 olhos $(18,5 \%)$, sendo que uma ocorreu por volta do $30^{\circ} \mathrm{PO}$, duas no $90^{\circ}$ e os outras duas ocorreram entre o $90^{\circ}$ e o $180^{\circ} \mathrm{PO}$.

\section{DISCUSSÃO}

A MA foi utilizada em cirurgias oculares pela primeira vez em 1940 por De Rotth ${ }^{(10)}$ no tratamento de simbléfaro e defeitos conjuntivais, sem resultados animadores, talvez pelo fato de ter sido usada a fresco e juntamente com o córion.

Foi visto que o córion é que possui atividade antigênica; no âmnion, a ausência de leucócitos facilita a realização de transplantes, não havendo chance de rejeição, sendo por isso indicada a utilização apenas do âmnion ${ }^{(11)}$.

A partir de 1995, os bons resultados com o uso da MA na forma preservada para o tratamento de úlceras corneanas induzidas em coelhos ${ }^{(5)}$, evidenciaram esse material como boa alternativa para tratamento de patologias da superfície ocular.

Quanto às formas de preservação, o meio mais utilizado atualmente é o meio de Eagle modificado por Dulbecco e glicerol $(1: 1)$, estocado a $-80^{\circ} \mathrm{C}$. Outras alternativas são a conservação com manutenção da membrana em glicerina à temperatura de $5^{\circ} \mathrm{C}^{(13)}$, ou ainda, a simples conservação à $-80^{\circ} \mathrm{C}$ para uso em período de 2 semanas $^{(1)}$.

O uso da MA no tratamento do pterígio recidivado foi explorado poucas vezes, mas os resultados relatados são animadores $^{(6)}$.

Todos os pacientes do presente estudo eram portadores de pterígio recidivado e aproximadamente $50,0 \%$ deles já haviam sido submetidos a 2 ou mais cirurgias. O prognóstico quando se opera pterígio recidivado é pior. No entanto, com o uso da MA observamos poucas complicações.

Com outros tratamentos adjuvantes também podem ocorrer problemas. O uso de betaterapia em pterígio recidivado pode levar a afinamento corneano, simbléfaro, catarata e ulceração corneana ${ }^{(12-13)}$. A mitomicina $C$, também usada para diminuir recidivas, pode levar a "melting" corneano ${ }^{(14)}$.

Com a MA, em todos os casos foi observada a presença de secreção esbranquiçada abundante até o $7^{\circ} \mathrm{PO}$. Acreditamos que essa secreção não esteja relacionada à infecção local, uma vez que a MA possui evidente ação antimicrobiana, além de ação anti-inflamatória ${ }^{(15)}$.

Com relação à reabsorção do material transplantado, percebemos que se trata de um processo rápido, tendo ocorrido, em todos os casos onde não houve perda do enxerto (25 olhos), até o $30^{\circ} \mathrm{PO}$. Em coelhos com úlcera corneana experimental, tratada com transplante de MA, a reabsorção mostrouse mais rápida do que em humanos, já que não foi identificada membrana nos cortes histopatológicos da córnea no $7^{\circ} \mathrm{PO}^{(16)}$.

A MA muitas vezes não se incorpora ao leito cruento mas sim, sofre dissolução, ocorrendo epitelização conjuntival subjacente, conforme já observado ${ }^{(17)}$.

Em nosso estudo observamos a formação de granuloma no local operado em $11,1 \%$ dos casos ( 3 olhos). Na literatura não há referências quanto a presença de granuloma e o uso de membrana amniótica e acreditamos que possam ter ocorrido devido ao fio de sutura utilizado para fixar a conjuntiva.

O índice de recidiva observado neste estudo foi de $18,5 \%$ (5 casos). Os índices de recidiva do pterígio citados na literatura são extremamente variáveis e dependem de diversos fatores dentre eles: amostra estudada, localização geográfica, tipo de atividade profissional do paciente, se a lesão é primária ou recidivada, tempo de seguimento, técnica cirúrgica empregada, uso de tratamentos coadjuvantes e mesmo a definição do que seria a recidiva. Todos estes fatores tornam muito difícil a comparação entre os estudos. Mesmo assim, pode-se dizer que os índices de recorrência com a técnica da esclera nua variam de 24 a $89 \%$. Com a aplicação de mitomicina haveria diminuição do número de recidivas de 0 a $38 \%$ e usando transplante de conjuntiva, as recidivas estariam entre 2 a $39 \% \%^{(18)}$.

Utilizando-se a MA, Shimazaki et al. ${ }^{(6)}$ tiveram $25,0 \%$ de recidiva, embora tenham estudado apenas 4 olhos operados.

Um fato curioso foi a ausência de recidiva nos dois casos em que houve a perda do enxerto de MA, uma vez que é conhecido que quando se utiliza técnica de rotação de retalhos, o índice de recorrência é maior se há retração do "flap"(19).

Comparando-se o transplante de MA com o auto-transplante de conjuntiva, outra técnica para tratamento do pterígio recidivado, percebemos que tecnicamente o transplante de MA é mais fácil de ser executado, com possibilidade de recorrência inferior ao observado com o transplante de conjuntiva ${ }^{(20)}$.

\section{CONCLUSÃO}

Apesar das complicações observadas e da possibilidade de nova recidiva da lesão, nossos resultados nos autorizam afirmar que o uso da MA no tratamento do pterígio recidivado é uma terapia efetiva e promissora, sendo uma boa alternativa nos casos onde o transplante conjuntival estaria contra-indicado, por exemplo em portadores de pterígio bilateral, em patologias conjuntivais sinequiantes e nos pacientes que futuramente necessitarão de cirurgia filtrante para tratamento do glaucoma.

\section{ABSTRACT}

Purpose: To evaluate the effectiveness of amniotic membrane transplantation for recurrent pterygium treatment. Methods: 
Amniotic membrane transplantation was performed in 27 eyes with recurrent pterygium, 7 with associated symblepharon. We evaluated age, sex, eye, site, number of previous surgeries and postoperative complications with a follow-up period of 6 months. Results: $58.3 \%$ of the patients were male and, $58.3 \%$ were between 40 and 59 years old. All of them had recurrent pterygium and $47.8 \%$ with at least two previous surgeries. We observed recurrence in $18.5 \%$ and $11.1 \%$ had granulomatous lesions. Conclusion: Amniotic membrane transplantation is a simple procedure and is a good choice to treat recurrent pterygium. However, this technique does not exclude the possibility to present lesion recurrence.

Keywords: Biological dressing/utilization; Pterygium/surgery; Amnion/transplantation; Ophthalmic surgical procedures

\section{REFERÊNCIAS}

1. Tsubota K, Satake Y, Ohyama M, Toda I, Takano Y, Ono M, Shinazaki N, Shimazaki I. Surgical reconstruction of the ocular surface in advanced ocular cicatricial pemphigoid and Stevens-Johnson syndrome. Am J Ophthalmol 1996;122:38-52.

2. Sorsby A, Haythorne J, Reed H. Amniotic Membrane grafts in caustic soda burns. Br J Ophthalmol 1947;31:401-4.

3. Shimazaki J, Yang, HY, Tsubota K. Amniotic Membrane transplantation for ocular surface reconstruction in patients with chemical and termal burns. Ophthalmology 1997;104:2068-76.

4. Tseng SC, Prabhassawat P, Lee SH. Amniotic Membrane Transplantation for conjunctival surface reconstruction. Am J Ophthalmol 1997;124:765-74.

5. Kim JC, Tseng SCG. Transplantation of preserved human amniotic membrane for surface reconstruction in severely damaged rabbit corneas. Cornea 1995; 14:473-84.

6. Shimazaki J. Shinozaki N, Tsubota K. Transplantation of amniotic membrane and limbal autograft for patients with recurrent pterygium associated with symblepharon. Br J Ophthalmol 1998;82:235-40.

7. Prabhasawa P, Barton K, Burkett G, Tseng SC. Comparison of conjunctival autografts amniotic membrane grafts and primary closure for pterigium excision. Ophthalmology 1997;104:974-85.

8. Fukuda K, Chikama T, Nakamura M, Nishida T. Differential distribution of subchains of the basement membrane components type IV collagen and laminin among the amniotic membrane, cornea and conjunctiva. Cornea 1999;18:73-9.

9. Adinolfi M, Akle CA, Mc Coll I, Fensom AH, Tansley L, Connolly P, et al. Expression of HLA antigens, B2-microglobulina and enzimes by human amniotic epithelial cells. Nature 1982;295:325-7.

10. De Roth. A plastic repair of conjunctival defects with fetal membrane. Arch Ophthalmol 1940;23:522-6.

11. Houlihan JM, Biro PA, Harper H, Jenskinson HJ, Holmes CH. The human amnion is a site of MHC Class 1b expression. J Immunol 1995;154:5665-74.

12. Oliveira PR de. Úlcera de esclera por beta-terapia após tratamento cirúrgico de pterígio. Rev Goiana Med 1985;31:167-70.

13. Dusenbery KE, Alul IH, Holland EJ, Khan FM, Levitt SH. B irradiation of recurrent pterygia-results and complications. Int J Radiat Oncol Biol Phys 1992;24:315-20.

14. Ewing-Chow DA, Romanchuk KG, Gilmour GR, Underhill JH, Climenhaga DB. Corneal melting after pterygium removal followed by topical mitomycin C therapy. Can J Ophthalmol 1992;27:197-9.

15. Talmi YP, Sigler L, Inge E, Finkelstein Y, Zohar Y. Antibacterial properties of human amniotic membranes. Placenta 1991;12:285-6.

16. Monteiro ECL, Schellini AS, Marques MEA, Kamegasawa A. Padovani CR.Tratamento da úlcera corneana experimental com membrana amniótica. Arq Bras Oftalmol 2000;63:33-7.

17. Gomes JAP, Komagome CM, Santos N, Chaves AP, Cunha MC, Freitas D. Membrana Amniótica nas cirurgias reconstrutivas da superfície ocular nas ceratoconjuntivites cicatriciais. Arq Bras Oftalmol 1999;62:562-76.

18. Sanches-Thorin JC, Rocha G, Yelin JB. Meta-analysis on the recurrence rates after bare sclera resection with and without mitomycin $\mathrm{C}$ use and conjunctival autograft placement in surgery for primary pterygium. Brit $\mathrm{J}$ Ophthalmol 1998;82:661-5.

19. McCoombes JA, Hirst LW, Isbell GP. Sliding Conjunctival flap for the treatment of primary pterygium Ophthalmology 1994;101:169-73.

20. Chen PP, Ariyasu RG, Kaza V, La Bree LD, Mc Donnell PJ. A randomized trial comparing mitomycin $\mathrm{C}$ and conjunctival autograft after excision of primary pterygium. Am J Ophthalmol 1995;120:151-60.

\title{
XXXVII Reunião Anual do Centro de Estudos Oftalmológicos "Cyro de Rezende"
}

\author{
27 e 28 de Outubro de 2001
}

Ribeirão Preto - SP

INFORMAÇÕES: Fax: (00xx16) 602-2860 